\title{
Association of diabetes-related autoantibodies with the incidence of asthma, eczema and allergic rhinitis in the TRIGR randomised clinical trial
}

\author{
Jeffrey P. Krischer $^{1}$ (D) David Cuthbertson ${ }^{1}$ (D) $\cdot$ Marisa Couluris $^{2} \cdot$ Mikael Knip $^{3,4}$ (D) $\cdot$ Suvi M. Virtanen $^{5,6,7,8}$ (D)
}

Received: 3 December 2019 / Accepted: 15 April 2020 / Published online: 17 June 2020

(C) Springer-Verlag GmbH Germany, part of Springer Nature 2020

\begin{abstract}
Aims/hypothesis This paper presents the relationship between islet autoantibodies, precursors of type 1 diabetes, and the development of persistent asthma, allergic rhinitis and atopic eczema.

Methods A total of 2159 newborns who had a first-degree relative with type 1 diabetes and selected HLA genotypes were followed until the youngest participant reached 10 years of age. Islet cell antibodies (ICA) were detected using indirect immunofluorescence. Autoantibodies to insulin (IAA), GAD (GADA), the tyrosine phosphatase-related insulinoma-associated 2 molecule (IA-2A) and zinc transporter 8 (ZnT8A) were quantified with the use of specific radiobinding assays. As an ancillary study, the incidence of asthma, allergic rhinitis and eczema was assessed in 1106 of these children using the International Study of Asthma and Allergies in Childhood (ISAAC) core questionnaire when the children were 9-11 years old. HRs with 95\% CIs were calculated to depict the incidence of these diseases following seroconversion to autoantibody positivity.

Results The cumulative incidence of atopic eczema, allergic rhinitis and persistent asthma were $22 \%, 9 \%$ and $7.5 \%$, respectively, by 9-11 years of age. The occurrence of diabetes-related autoantibodies showed a protective association with subsequently reported incidence of asthma and eczema. The incidence of rhinitis was not significantly related to the occurrence of IAA or GADA (statistical power was limited), but demonstrated the same inverse relationship as did the other diseases with ICA or when multiple autoantibodies first appeared together.

Conclusions/interpretation The findings add evidence to the relationships between these atopic diseases and diabetes-related autoimmunity and also suggest that, for eczema, the interaction depends upon which autoantibody appeared first.

Trial registration ClinicalTrials.gov NCT00179777
\end{abstract}

Keywords Asthma $\cdot$ Autoimmunity $\cdot$ Eczema $\cdot$ Rhinitis $\cdot$ Type 1 diabetes

\section{Abbreviations}

GADA GAD autoantibodies

IA-2A Tyrosine phosphatase-related insulinoma-associated 2 molecule autoantibodies

IAA Insulin autoantibodies

ICA Islet cell antibodies

ISAAC International Study of Asthma and Allergies in Childhood

Jeffrey P. Krischer

jeffrey.krischer@epi.usf.edu

Extended author information available on the last page of the article
TRIGR Trial to Reduce Insulin Dependent Diabetes in the Genetically at Risk

ZnT8A Zinc transporter 8 antibodies

\section{Introduction}

The prevalence rates of asthma and other allergic diseases in children share many of the same characteristics as that of type 1 diabetes and its associated autoimmunity. Both have been increasing in prevalence during the last six decades, particularly in industrialised countries, although the increase may have levelled off in some [1-4].

Allergic diseases are caused by over-reactivity of the human immune system against foreign proteins, leading to 


\section{Research in context}

\section{What is already known about this subject?}

- Prevalence rates of type 1 diabetes and its associated autoimmunity, as well as asthma and allergic diseases in children, have been increasing during the last six decades

\section{What is the key question?}

- What was the relationship between the incidence of diabetes-related autoantibodies and the development of persistent asthma, allergic rhinitis and atopic eczema in the TRIGR study?

\section{What are the new findings?}

- Occurrence of diabetes-related autoantibodies showed a protective association with subsequently reported incidence of asthma and eczema

- Incidence of rhinitis was not significantly related to the occurrence of the autoantibodies IAA or GADA, but demonstrated the same inverse relationship as did the other diseases with ICA or when multiple autoantibodies first appeared together

\section{How might this impact on clinical practice in the foreseeable future?}

- These findings suggest an interaction between these atopic diseases and diabetes-related autoimmunity

asthma, allergic rhinitis, conjunctivitis, atopic eczema and food allergies. Parental heredity is a strong risk factor for the development of both asthma and atopic eczema: if one of the parents has the allergic disease the risk is increased at least twofold; if both, the increase is three- to fourfold $[5,6]$. However, the rapid increase in the occurrence of these diseases over time indicates that environmental factors are also crucial in the disease aetiology. The same picture is found in diabetes-related autoimmunity and type 1 diabetes. A family history of type 1 diabetes increases diabetes risk and there is substantial evidence of the role of environmental exposures [7].

The immune abnormalities that lead to allergic diseases are initiated in infancy and possibly even during the fetal period. Diabetes-related autoimmunity has also been shown to occur in infancy for those presenting with insulin autoantibodies (IAA) as their first-appearing autoantibody, but at a later age for those presenting with GAD autoantibodies (GADA) first $[8,9]$. Thus, early months of development may be critical for the maturation of the immune system as well as complex immune regulatory networks that result in functional disturbances, autoimmunity and allergic disorders.

The nutritional factors identified in epidemiological studies as possibly affecting the development of islet cell autoimmunity and allergic diseases and asthma include breastfeeding, age at introduction of new foods in infancy, dietary fatty acids, dietary antioxidants, vitamin $\mathrm{D}$, foods that affect gut microbiota and obesity $[10,11]$. There is contrasting evidence of whether prolonged feeding with a hydrolysed infant formula compared with a formula based on intact cow's milk reduces infant and childhood allergies or diabetes-related autoimmunity [12-14].
This paper explores the relationship between the incidence of asthma, rhinitis and eczema and the incidence of diabetesrelated autoantibodies in a subset of the children enrolled in the Trial to Reduce Insulin Dependent Diabetes in the Genetically at Risk (TRIGR) study (ClinicalTrials.gov Identifier: NCT00179777). The TRIGR infant feeding trial was designed to examine whether weaning to an extensively hydrolysed infant formula, as compared with formula based on cow's milk, delays or prevents the development of islet cell autoimmunity and/or type 1 diabetes in children at increased genetic risk of type 1 diabetes. TRIGR was a double-blind, randomised controlled trial of a dietary intervention (hydrolysed vs standard infant formula) that enrolled 2159 newborn first-degree relatives of patients with type 1 diabetes from 77 clinical sites in 15 countries [15-17]. The intervention period concluded in August 2007. This cross-sectional asthma and allergy ancillary study was performed between 2013 and 2016, when the children were 9-11 years old.

\section{Methods}

Participants Newborn infants who had a first-degree relative with type 1 diabetes and defined HLA genotypes were recruited into TRIGR between May 2002 and January 2007, and followed until diagnosis of type 1 diabetes or until the youngest participant reached 10 years of age in 2017 . Randomisation of the infants who met the inclusion criteria took place before birth or immediately after birth. Altogether, 2159 newborn infants (1142 boys [52.9\%]) with an eligible HLA genotype (41.9\% of the genotyped infants) were randomised to the TRIGR intervention study. Randomisation 
resulted in balanced groups with 1081 infants in the casein hydrolysate group and 1078 in the cow's milk group. In total, 516 infants $(23.9 \%)$ carried the high-risk HLA genotype, 953 (44.1\%) carried moderate-risk genotypes, 668 (30.9\%) carried mild-risk genotypes and $22(1.0 \%)$ carried the rare mild-risk genotype (Table 1). The first-degree relative with type 1 diabetes was the mother in 1055 infants (48.9\%), the father in 723 $(33.5 \%)$ and a sibling in $308(14.3 \%)$, whereas 73 participants (3.4\%) had multiple affected relatives. The median follow-up time for the diagnosis of diabetes was 11.5 years (range 3 months to 14.8 years; mean 11.0 years).

Written, informed consent was obtained from the family before enrolment into the TRIGR study. The study was approved by the ethics committees of all participating centres.

Dietary intervention Infants were randomly assigned weaning to either the intervention or control formula, produced specifically for this study. The intervention formula was an extensively hydrolysed casein-based formula, while the control formula was composed of $80 \%$ intact cow's milk protein and $20 \%$ hydrolysed milk protein, and formulated so that the taste and smell would be indistinguishable from the intervention formula. Study formulas were prepared and coded with the use of four colours by Mead Johnson Nutrition and were blinded to all investigators except the Data Management Unit (DMU). Newborn infants requiring supplemental feeding before randomisation (e.g., infants born at night or on weekends) received banked breast milk or Nutramigen, an extensively hydrolysed casein-based formula.

Breastfeeding was practiced at the discretion of the participating mothers, and maternal diets were unmodified. Breastfeeding was encouraged and exceeded national averages in both groups [18]. The dietary intervention period lasted until the infant was at least 6 months of age, and if by that time the child had not received the study formula for at least 60 days, study formula feeding was continued until 60 days of study formula exposure was reached, but not beyond 8 months of age. Parents were asked not to feed the children any commercial or other baby foods containing bovine protein during the intervention period. Adherence to the protocol was monitored by means of regular family nutrition interviews (at the ages of $0.5,1,2,3,4,5,6,7$ and 8 months) and by the analysis of cow's milk antibodies in serum samples. Detailed study design and methods have been previously published [16-18]. Online informed consent for this ancillary study was obtained for all study participants from a parent or primary caretaker.

HLA genotyping Cord blood or a heel stick blood sample collected on filter paper shortly after birth was immediately sent to the Turku (Europe and Australia) or Pittsburgh (North America) laboratory for HLA genotyping. HLA genotyping for the selected $D Q B 1$ and $D Q A 1$ alleles was performed using sequence-specific oligonucleotide hybridisation, with quality control between the two laboratories carefully maintained. The following genotypes were regarded as eligible: (1) $H L A$ $D Q B 1 * 02 / D Q B 1 * 03: 02$ [high-risk]; (2) HLA DQB1*03:02/x (x not $D Q B 1 * 02, D Q B 1 * 03: 01$ or $D Q B 1 * 06: 02$ ) [moderate risk]; (3) $H L A D Q A 1 * 05-D Q B 1 * 02 / y$ (y not $D Q A 1 * 02: 01$ $D Q B 1 * 02, D Q B 1 * 03: 01, D Q B 1 * 06: 02$ or $D Q B 1 * 06: 03)$ [mild risk]; and (4) HLA DQA1*03-DQB1*02/y (y not $D Q A 1 * 02: 01-D Q B 1 * 02, D Q B 1 * 03: 01, D Q B 1 * 06: 02$ or $D Q B 1 * 06: 03)$ [rare mild risk].

Diabetes-related autoantibodies Blood samples for autoantibody analyses were obtained (after application of analgesic cream) at the follow-up visits when the children were 3,6 , 9, 12, 18 and 24 months of age, and annually thereafter up to the age of 10-14 years. Serum samples were stored at $-70^{\circ} \mathrm{C}$ until they could be analysed. Islet cell antibodies (ICA) were detected with the use of indirect immunofluorescence, while the four biochemical autoantibodies (IAA, GADA, autoantibodies to the tyrosine phosphatase-related insulinoma-associated 2 molecule [IA-2A] and autoantibodies to zinc transporter 8 [ZnT8A]) were quantified with the use of specific radiobinding assays [19]. Each autoantibody except for ZnT8A was analysed from all samples available. ZnT8A was initially quantified from the samples taken at the ages of $2,3,4,6,8,10$ and 12 years. If the 2 year sample tested positive, the samples obtained at the ages of $3,6,8,9,12$ and 18 months were also analysed to assess the age at seroconversion. Similarly, any later samples between a negative and a positive sample were analysed.

Study endpoints The incidence of asthma, allergic rhinitis and eczema was assessed using the standardised and validated International Study of Asthma and Allergies in Childhood (ISAAC) core questionnaire on the child's history presented online to the parents of the participating children [20]. The online assessment included additional questions related to the child's use of asthma medications (if applicable), whether the child was ever diagnosed by a physician with certain diseases and food allergies, exposure to possible allergens and other factors related to asthma and/or allergies. Persistent asthma was defined as doctor-diagnosed asthma plus either any wheezing symptom during the preceding 12 months or use of asthma medication during the preceding 12 months. Age at onset of asthma was determined by the question: 'At what age did the symptom of asthma start or at what age did the child first wheeze?' Wheezing was defined as any of the following during the past 12 months: wheezy sound in respiration; wheezy sound during respiration in association with physical activity; difficulties in respiration in the morning on waking up; or wheezy respiration without having sniffles or respiratory infection. Allergic rhinitis was defined as doctor-diagnosed hay fever or allergic rhinoconjunctivitis 


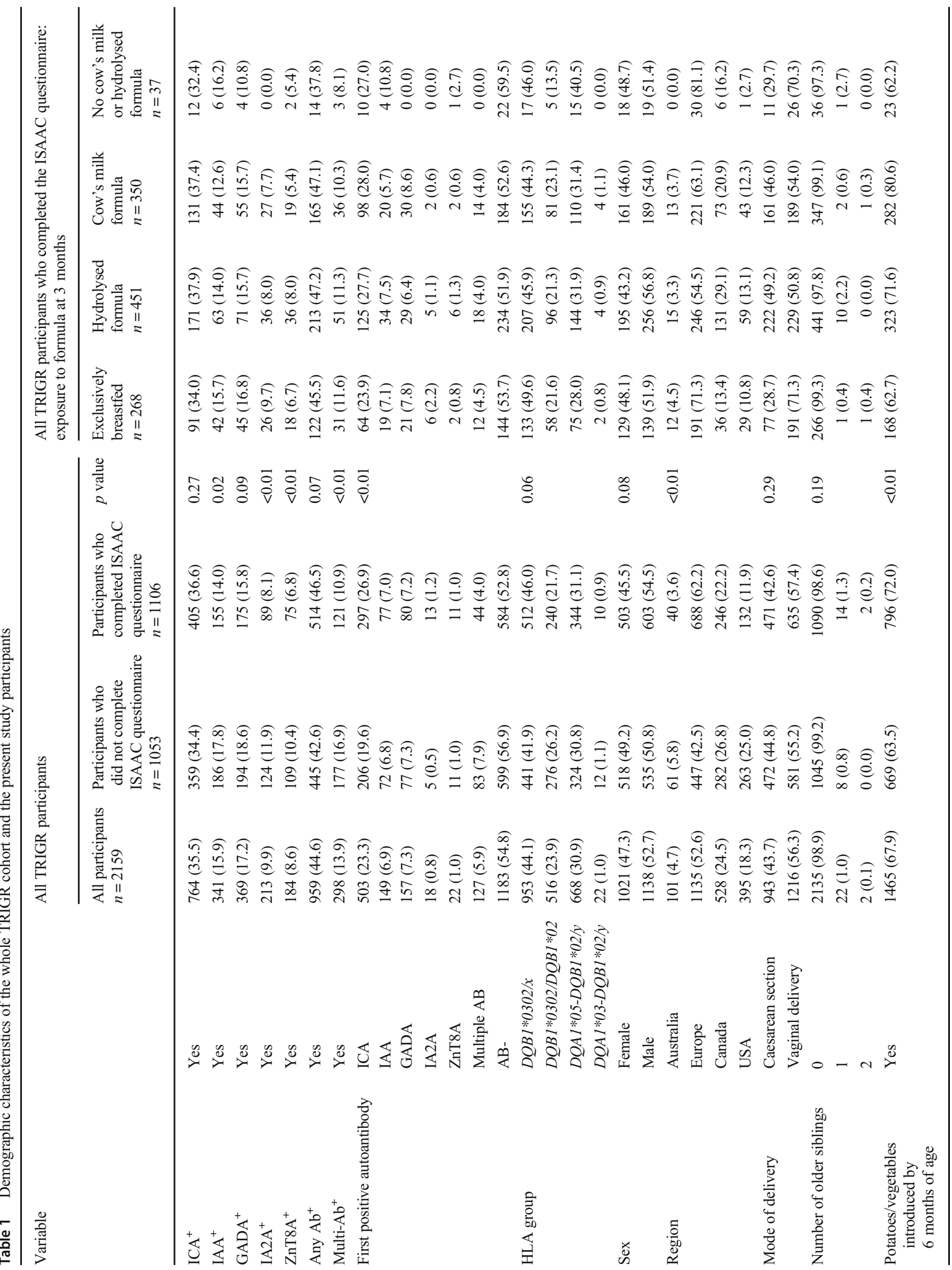




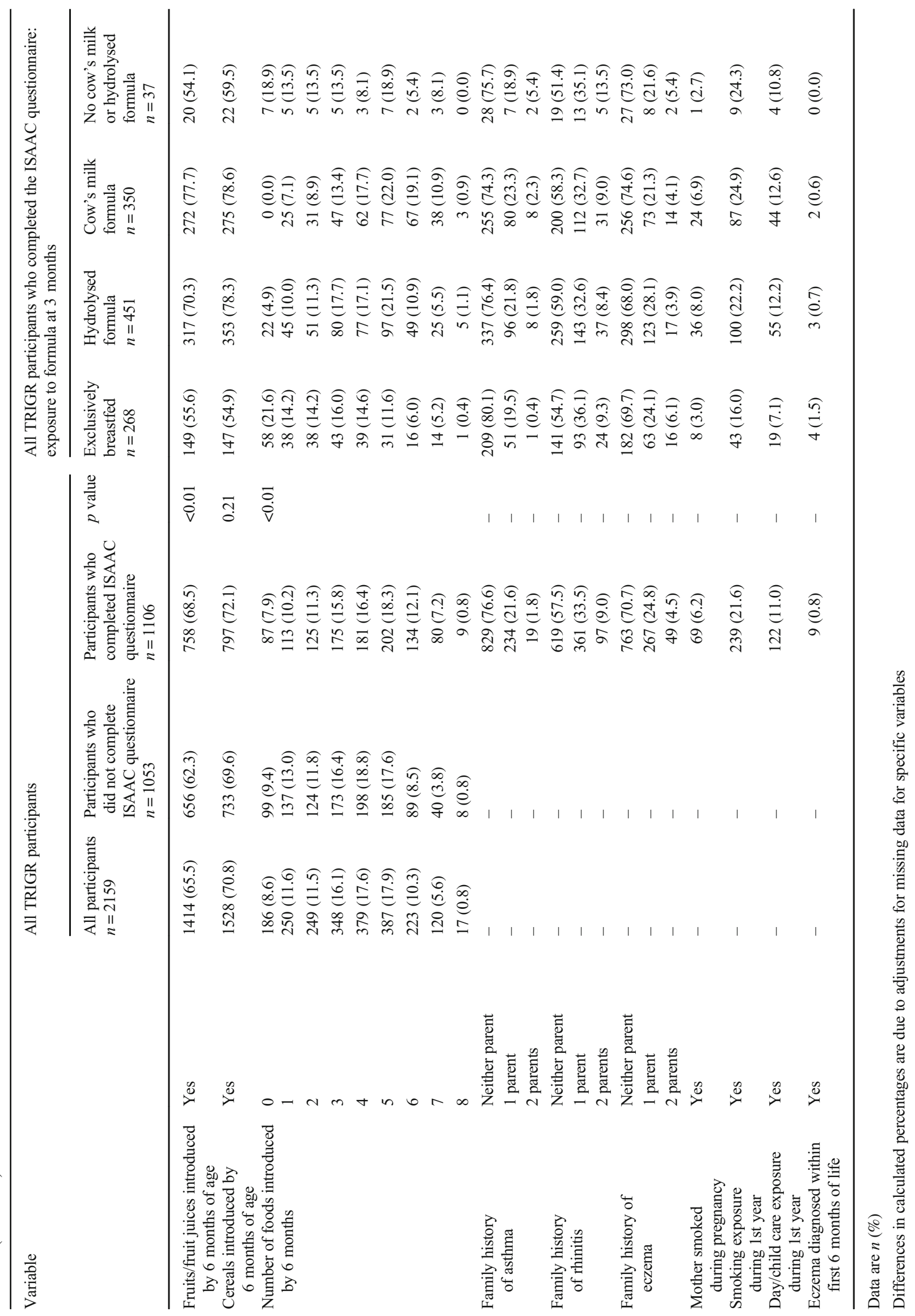


during the preceding 12 months. Age at onset of allergic rhinitis was based on the age of the child at the onset of symptoms including sneezing, nasal congestion or rhinitis other than with respiratory infections, accompanied by itching of the eyes and tearing. Atopic eczema was defined as ever having doctor-diagnosed atopic eczema. Age of onset of atopic eczema was based on the question: 'At what age did the child first have atopic eczema?' All online questionnaires, including ISAAC, were available in local languages.

Statistical analyses The cumulative incidence of onset of each endpoint from birth was estimated using a modified KaplanMeier disease-free survival function. The differences between groups in the cumulative incidence functions, and the associated hazard functions, were tested using the logrank test according to the actual diet consumed. The relative risk of disease onset between groups was estimated with the Cox proportional hazards model using time-dependent covariates. Multivariate analyses included characteristics that were found to be statistically significantly associated with autoantibody incidence from the univariate analyses. The autoantibodynegative group was defined to be the referent population for comparing disease incidence by first-appearing autoantibody. When comparing characteristics between the extensively hydrolysed formula and cow's milk formula groups, the $t$ test was applied for normally distributed variables and the nonparametric Mann-Whitney $U$ test for skewed variables.

Although the overall study sample size was substantial, the statistical power for testing the effect of covariates on each disease was limited due to the number of children having had that diagnosis. The TRIGR study had $>80 \%$ power to detect HRs of 0.5 or less in autoantibody incidence according to the use of hydrolysed formula. The power to detect differences in the incidence of atopic diseases, which occurred with lower frequency, conditioned upon autoantibody occurrence was much less. The detectable HRs at $80 \%$ power ranged from 0.1 to 0.3 for asthma, allergic rhinitis and eczema.

Data were analysed using the Statistical Analysis System software (version 9.4; SAS Institute, Cary, NC, USA). Twotailed $p$ values less than 0.05 were considered to be statistically significant. No adjustment in type 1 error was made for multiple comparisons except in the context of the multiple Cox regression model.

The TRIGR study and this ancillary study was approved by local institutional review or ethics boards and was monitored by an External Evaluation Committee formed by the National Institutes of Health.

\section{Results}

Of the total study population, $1106(51.2 \%)$ completed the ISAAC questionnaire and were included in this analysis.
Altogether, 268 children in this asthma and allergy study population were exclusively breastfed at 3 months of age, 451 received hydrolysed formula, 350 received cow's milk formula and the remaining 37 were not exclusively breastfed but did not receive either of the formulas. There were statistically significant differences between the enrolled total TRIGR cohort and those who completed the ISAAC questionnaire according to their demographic characteristics. A higher proportion of the ISAAC responders were enrolled from TRIGR European countries as compared with North America or Australia ( $62 \%$ vs $43 \%, p<0.01)$, had an increased number of foods introduced by 6 months of age $(20 \%$ vs $13 \%, p<0.01)$ (notably potatoes/vegetables $[72 \%$ vs $64 \%$ ] and fruits/fruit juices [69\% vs 62\%]) and were exclusively breastfed for the first 3 months of life (24\% vs $18 \%$, $p<0.01)$. With regard to other demographics measured, there were no statistically significant differences between those who responded to the ISAAC questionnaire and those who did not.

Comparing feeding practices during the first 3 months of life for those who completed the ISAAC questionnaire, among those exclusively breastfed or that did not receive hydrolysed or cow's milk-containing formulas, a higher percentage of respondents were from sites in Europe as compared with the remaining TRIGR sites $(72.5 \%$ vs $58.3 \%, p<0.001)$ or were delivered vaginally $(71.1 \%$ vs $52.2 \%, p<0.001)$, and a lower percentage had diets that included potatoes/vegetables $(62.6 \%$ vs $75.5 \%)$, fruits/fruit juices $(55.4 \%$ vs $73.5 \%)$ and cereals $(55.4 \%$ vs $78.4 \%)$ by 6 months of age (all $p<0.0001)$. Also, respondents who were exclusively breastfed or did not receive hydrolysed or cow's milk-containing formulas were less likely to come from families where mothers smoked during pregnancy $(3.0 \%$ vs $7.5 \%$, $p=0.005$ ) or whose children lived in a household with an active smoker during the first year of life (17.0\% vs $23.3 \%$, $p=0.023)$, as compared with the other feeding groups (Table 1).

The cumulative incidence rates for eczema, allergic rhinitis and asthma were $22 \%(N=251$ [95\% CI 18.5\%, 25.7\%]), 9\% $(N=112[95 \% \mathrm{CI} 6.8 \%, 11.2 \%])$ and $7.5 \%(N=89[95 \% \mathrm{CI}$ $3.8,11.2]$ ), respectively, by $9-11$ years of age (Fig. 1). Among those who remained autoantibody negative $(N=599)$, the cumulative incidence rates of eczema, allergic rhinitis and asthma were $33 \%$ (95\% CI 29.3\%, 36.9\%), 14\% (95\% CI $10.8 \%, 17.8 \%)$ and $12.4 \%(95 \%$ CI $9.7 \%, 15.1 \%)$ by $9-$ 11 years of age. These rates are lower than anticipated from the ISAAC study conducted in these same countries, but not by much when considering those who remained autoantibody negative through the follow-up period. Among the TRIGR countries, the prevalence of asthma in children between 6 and 14 years of age as defined by the ISAAC study was highest in Australia (26-37\% for asthma). For the USA, the prevalence was 17-20\%, and in Canada it was 16-19\%. The prevalence was lowest among European countries 
Fig. 1 Cumulative incidence of persistent asthma, atopic eczema and allergic rhinitis

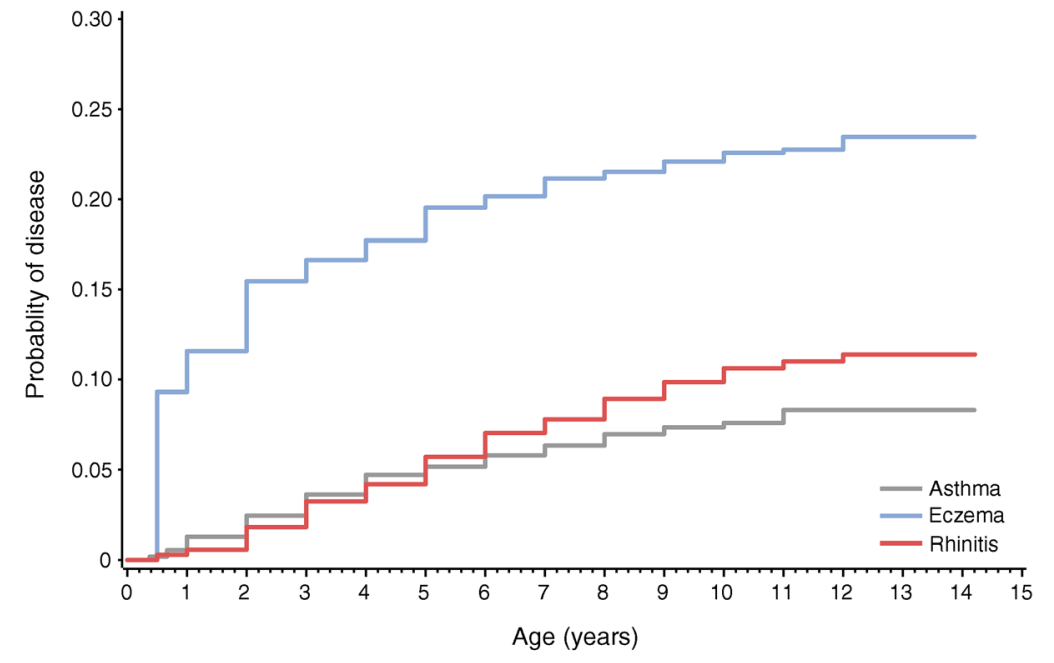

Number at risk:

$\begin{array}{llllllllllllllll}\text { Asthma } & 1104 & 1090 & 1077 & 1064 & 1052 & 1047 & 1040 & 1034 & 1027 & 960 & 754 & 515 & 249 & 86 & 16\end{array}$

$\begin{array}{llllllllllllllll}\text { Eczema } & 1106 & 978 & 935 & 922 & 910 & 890 & 883 & 872 & 868 & 814 & 636 & 437 & 213 & 70 & 15\end{array}$

$\begin{array}{llllllllllllllll}\text { Rhinitis } & 1051 & 1045 & 1032 & 1017 & 1007 & 991 & 977 & 969 & 957 & 896 & 697 & 468 & 227 & 84 & 16\end{array}$ participating in TRIGR, ranging from the lowest estimates in Estonia (4-5\%) to the highest in Italy (11-15\%). The prevalence rates of current wheezing according to ISAAC in children of 6-14 years old were: Australia 20-31\%, USA 19$22 \%$, Canada $14-15 \%$, Estonia $9-10 \%$ and Italy $8-11 \%$. The prevalence rates of allergic sensitisation to any allergen across the TRIGR countries were in the range of $35-40 \%$ [19-23].

Disease risk was significantly increased $(p<0.01)$ for each of the three diseases with a family history of that disease. Also, there was a significantly higher risk for asthma (HR 81.02, $p<0.01$ ) if eczema was diagnosed 6 months earlier or within the first 6 months of age (HR 2.37, $p=0.002$ ). Excluding IA$2 \mathrm{~A}$ and ZnT8A due to small numbers ( $N=8$ for each), the development of islet cell autoimmunity was significantly associated with a reduced subsequent risk of asthma or eczema, no matter which autoantibody was present, with HRs ranging from 0.13 to 0.18 (Table 2, Fig. 2). The presence of ICA $(N=233$, HR $0.41, p<0.01)$ significantly reduced the risk of allergic rhinitis, but neither IAA $(N=39)$ nor GADA $(N=48)$ had a significant impact on allergic rhinitis (HR $0.63, p=0.14$ and HR $0.57, p=0.09$, respectively). ICA, as the first-appearing autoantibody $(N=233)$, significantly reduced the subsequent risk of asthma (HR $0.22, p<0.01)$, rhinitis (HR 0.40, $p<0.01$ ) and eczema (HR 0.27, $p<0.01$ ). IAA or GADA as the first-appearing autoantibody was followed by a significant risk reduction only for eczema (HR $0.29, p=0.01$ and HR $0.33, p=0.02$, respectively). The only other significant finding was the lower risk (HR 0.55, $p=$ 0.01) of asthma for study children from Europe as compared with children from the USA.

Multivariable analyses of these risk factors resulted in consistent statistically significant reduced risk when ICA was the first-appearing autoantibody $(p<0.01$; HR 0.24 , HR
0.40 and HR 0.27 for asthma, allergic rhinitis and eczema, respectively). When multiple autoantibodies appeared first $(N=169)$, the reduced risk remained significant for asthma (HR 0.06, $p<0.01$ ) and allergic rhinitis (HR $0.38, p<0.01$ ), but the risk reduction was no longer significant for eczema $(p<0.12)$. For eczema, IAA and GADA, as first-appearing autoantibodies, were each associated with a statistically significant risk reduction ( $\mathrm{HR} 0.30, p=0.02$ and HR 0.34, $p=0.02$, respectively). The appearance of these autoantibodies individually did not result in a statistically significant impact on risk for asthma or allergic rhinitis. Family history (either one or both parents) conveyed a significantly higher risk across all three diagnoses, as did a family history of eczema for asthma, similar to what was seen in the univariate analyses (Table 3).

\section{Discussion}

The lower reported incidence of persistent asthma, allergic rhinitis and eczema in the TRIGR countries could be explained, in part, by the higher incidence of diabetesrelated autoantibodies in the TRIGR study population and the inverse relationship of their incidence found in this study. This finding is consistent with other studies in North America [24] and Europe [25] that report a lower prevalence of asthma in children with type 1 diabetes, as compared with the general population; however, some findings have differed, especially the USA [26]. Hence, the relationship between type 1 diabetes and atopic disease remains controversial. That the TRIGR study cohort was not population based, but selectively included children at higher genetic risk for diabetes among families with a first-degree relative with type 1 diabetes, could be considered a limitation on the findings reported herein. However, this paper focuses on the relationship between 


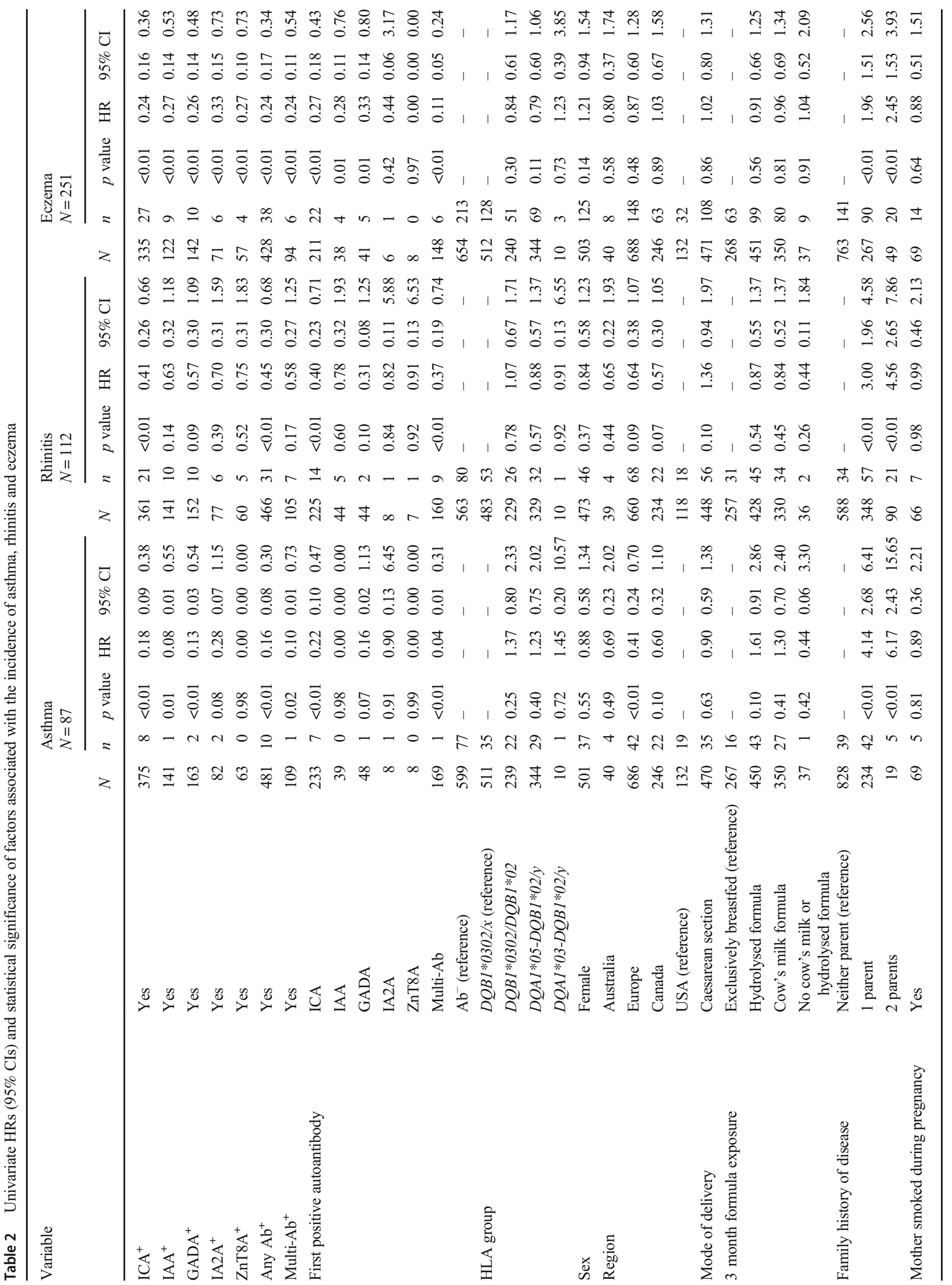




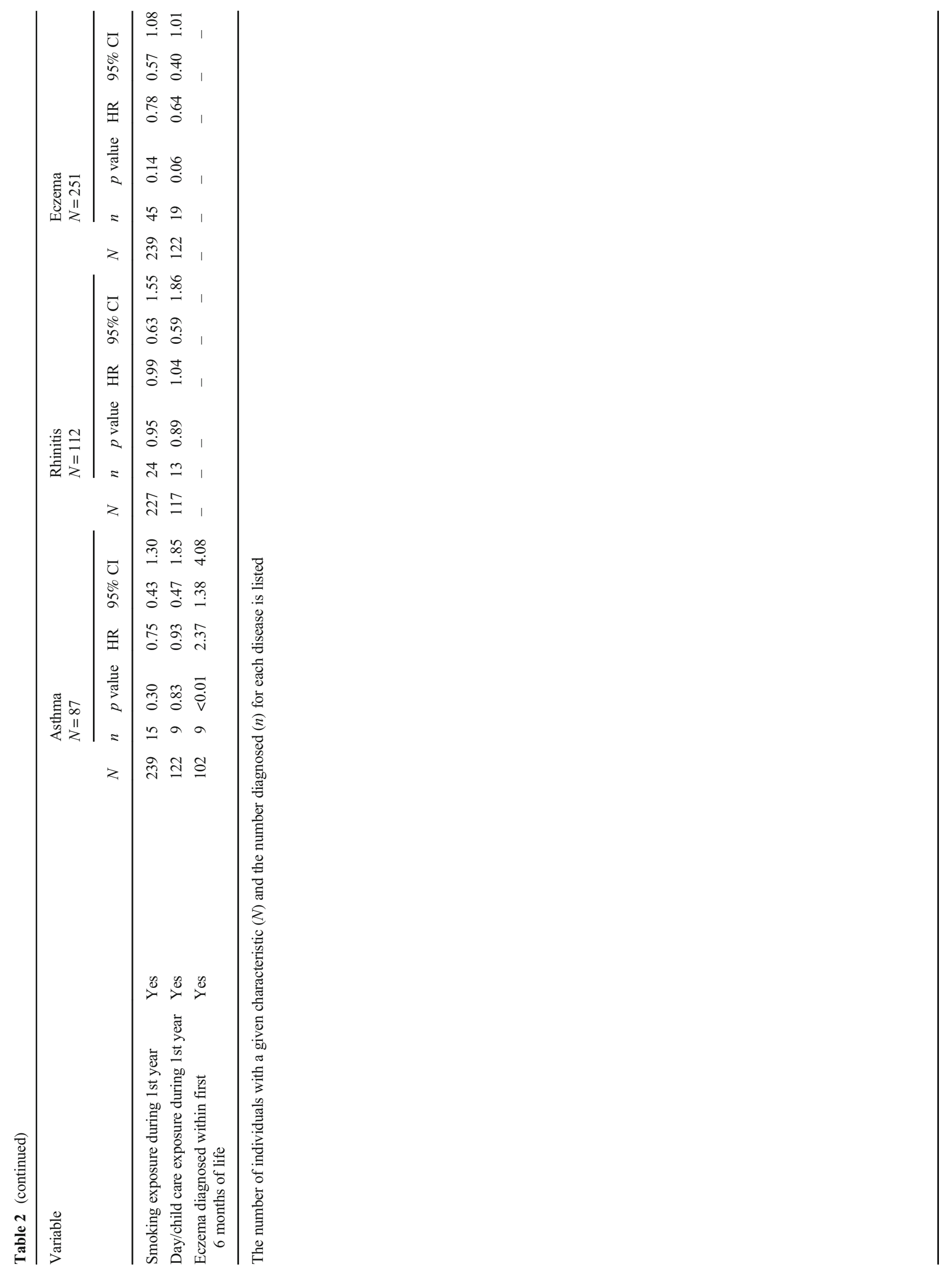


a

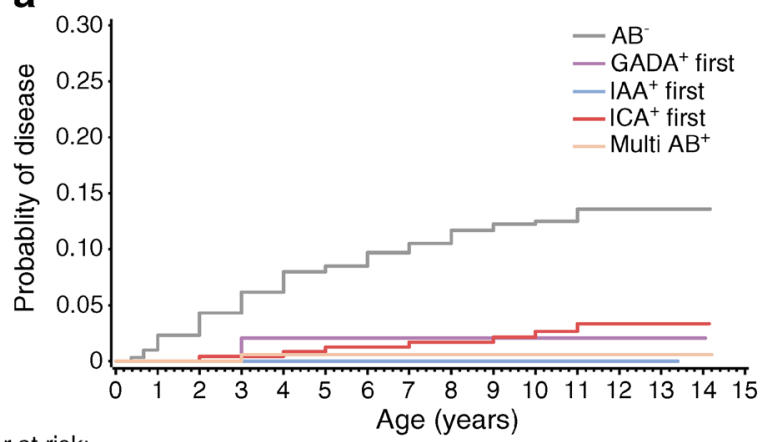

b

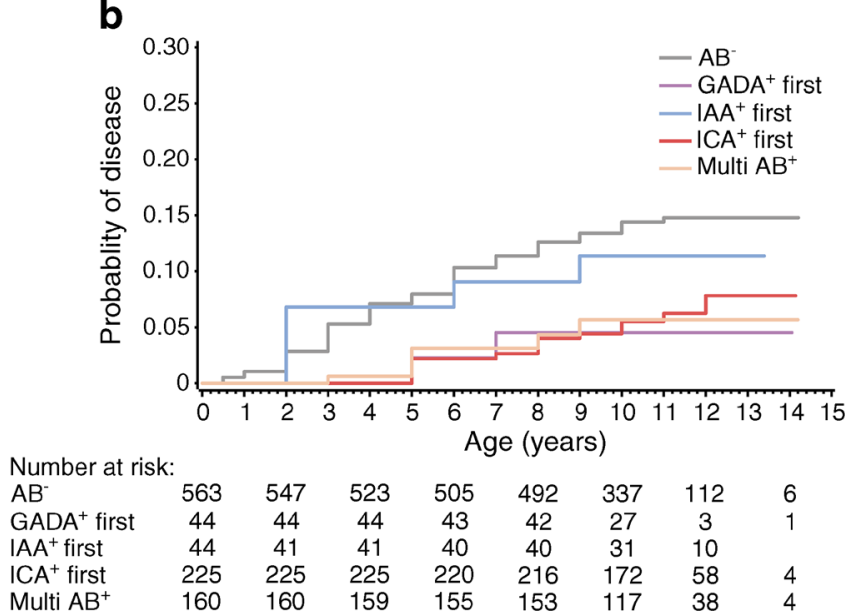

Number at risk:

$\begin{array}{lcccccccc}\mathrm{AB}^{-} & 563 & 547 & 523 & 505 & 492 & 337 & 112 & 6\end{array}$

$\mathrm{AA}^{+}$first

$\mathrm{ICA}^{+}$first

Multi $\mathrm{AB}^{+}$

$\begin{array}{cccccccc}44 & 44 & 44 & 43 & 42 & 27 & 3 & 1 \\ 44 & 41 & 41 & 40 & 40 & 31 & 10 & \\ 225 & 225 & 225 & 220 & 216 & 172 & 58 & 4 \\ 160 & 160 & 159 & 155 & 153 & 117 & 38 & 4\end{array}$

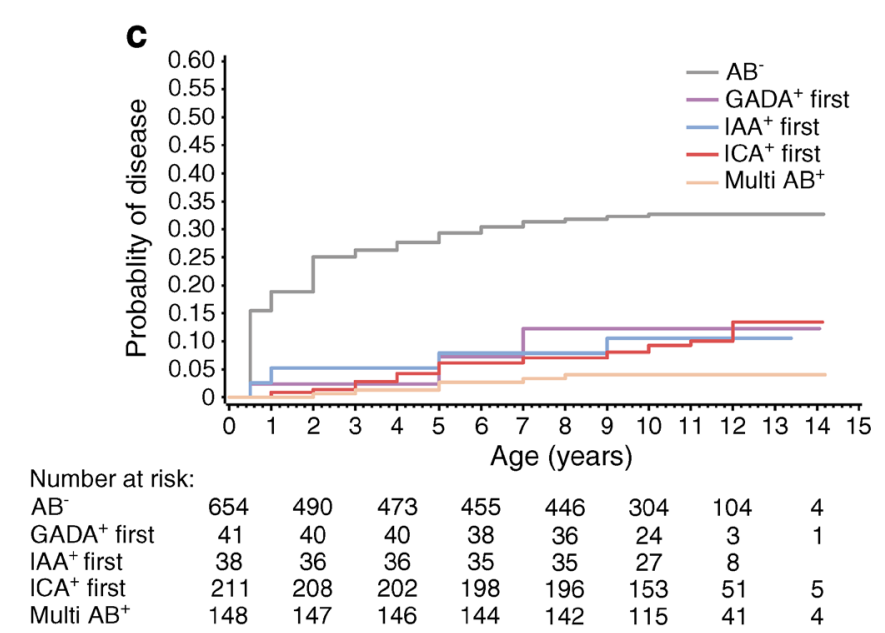

Fig. 2 Cumulative incidence by prior autoantibody status for asthma (a), eczema (b) and allergic rhinitis (c)

autoimmunity and asthma, allergic rhinitis and eczema, not the prevalence of these disorders. The selection bias would be pertinent only if one thought that the relationship was different among those who developed diabetes-related autoimmunity and these atopic diseases in other, lower risk, HLA groups. There are no reports in the literature to support this contention.

The occurrence of diabetes-related autoantibodies showed a protective association with subsequent development of persistent asthma and atopic eczema. The incidence of allergic rhinitis was not significantly related to the occurrence of IAA or GADA (statistical power was limited), but demonstrated the same inverse relationship, as did the other diseases, with ICA or when multiple autoantibodies first appeared together. Hence, when considering putative confounders and all of the first-appearing endpoints simultaneously, ICA and multiple autoantibodies were associated with persistent asthma and allergic rhinitis, and ICA, IAA and GADA with atopic eczema. The lack of statistical significance in the comparison of effect of the occurrence of IAA or GADA with allergic rhinitis must be interpreted with some caution as the study has limited statistical power, as noted above.
These findings extend the observation of an inverse relationship between type 1 diabetes and later development of asthma, showing the same directional effect but suggesting that the protective effect extends to the first appearance of autoantibodies [27]. As far as we are aware, this has not been shown before. An earlier study has observed a direct association between wheezing during the first year of life and presence of GADA and IA-2A autoimmunity at the age of 2.5 years [28]. The present study did not observe sufficient numbers of cases of seroconversion to autoantibody positivity after a diagnosis of asthma or allergic rhinitis to test the question of whether earlier diagnosis of these diseases increased the incidence of autoimmunity. In this population, autoantibodies most often occurred in early childhood, preceding disease diagnosis, except for eczema, which was diagnosed at a very early age. In this study, islet autoantibodies were rarely seen before 1 year of age. In other studies [8], when they are seen, IAA is most often the first-appearing autoantibody. Also, this study did not observe a difference in disease diagnosis according to the sex of the child. The random allocation to different early feeding patterns also supports the lack of an observed 
Table 3 Multivariate HRs (95\% CIs) and statistical significance of factors affecting the incidence of asthma, rhinitis and eczema

\begin{tabular}{|c|c|c|c|c|}
\hline Disease & $p$ value & HR & \multicolumn{2}{|c|}{$95 \% \mathrm{CI}$} \\
\hline \multicolumn{5}{|l|}{ Asthma $(n=87)$} \\
\hline \multicolumn{5}{|l|}{ First positive autoantibody } \\
\hline ICA & $<0.01$ & 0.21 & 0.10 & 0.46 \\
\hline IAA & 0.98 & 0.00 & 0.00 & 0.00 \\
\hline GADA & 0.05 & 0.14 & 0.02 & 1.03 \\
\hline $\mathrm{IA} 2 \mathrm{~A}$ & 0.82 & 0.79 & 0.11 & 5.96 \\
\hline ZnT8A & 0.99 & 0.00 & 0.00 & 0.00 \\
\hline Multi-Ab & $<0.01$ & 0.05 & 0.01 & 0.37 \\
\hline $\mathrm{Ab}^{-}$(reference) & & - & - & - \\
\hline \multicolumn{5}{|l|}{ Region } \\
\hline Australia & 0.29 & 0.55 & 0.18 & 1.66 \\
\hline Europe & 0.02 & 0.53 & 0.31 & 0.92 \\
\hline Canada & 0.26 & 0.70 & 0.38 & 1.30 \\
\hline USA (reference) & & - & - & - \\
\hline \multicolumn{5}{|l|}{ Family history of disease } \\
\hline Neither parent (reference) & & - & - & - \\
\hline 1 parent & $<0.01$ & 3.82 & 2.46 & 5.92 \\
\hline 2 parents & $<0.01$ & 6.80 & 2.55 & 18.14 \\
\hline \multicolumn{5}{|c|}{ Eczema diagnosed within first 6 months of life } \\
\hline Yes & $<0.01$ & 2.23 & 1.29 & 3.86 \\
\hline \multicolumn{5}{|l|}{ Rhinitis $(n=112)$} \\
\hline \multicolumn{5}{|l|}{ First positive autoantibody } \\
\hline ICA & $<0.01$ & 0.40 & 0.23 & 0.71 \\
\hline IAA & 0.68 & 0.83 & 0.34 & 2.04 \\
\hline GADA & 0.06 & 0.26 & 0.06 & 1.05 \\
\hline $\mathrm{IA} 2 \mathrm{~A}$ & 0.89 & 0.87 & 0.12 & 6.28 \\
\hline ZnT8A & 0.84 & 0.82 & 0.11 & 5.90 \\
\hline Multi-Ab & $<0.01$ & 0.38 & 0.19 & 0.75 \\
\hline $\mathrm{Ab}^{-}$(reference) & & - & - & - \\
\hline \multicolumn{5}{|l|}{ Family history of disease } \\
\hline Neither parent (reference) & & - & - & - \\
\hline 1 parent & $<0.01$ & 2.98 & 1.95 & 4.56 \\
\hline 2 parents & $<0.01$ & 4.70 & 2.72 & 8.10 \\
\hline \multicolumn{5}{|l|}{ Eczema $(n=251)$} \\
\hline \multicolumn{5}{|l|}{ First positive autoantibody } \\
\hline ICA & $<0.01$ & 0.27 & 0.18 & 0.43 \\
\hline IAA & 0.01 & 0.29 & 0.11 & 0.77 \\
\hline GADA & 0.02 & 0.34 & 0.14 & 0.83 \\
\hline $\mathrm{IA} 2 \mathrm{~A}$ & 0.45 & 0.47 & 0.07 & 3.36 \\
\hline ZnT8A & 0.97 & 0.00 & 0.00 & 0.00 \\
\hline Multi-Ab & $<0.01$ & 0.11 & 0.27 & 0.18 \\
\hline $\mathrm{Ab}^{-}$(reference) & & - & - & - \\
\hline \multicolumn{5}{|l|}{ Family history of disease } \\
\hline Neither parent (reference) & & - & - & - \\
\hline 1 parent & $<0.01$ & 1.79 & 1.38 & 2.34 \\
\hline 2 parents & $<0.01$ & 2.14 & 1.34 & 3.43 \\
\hline
\end{tabular}

relationship between atopic disease incidence and early exposure to hydrolysed formula compared with cow's milk-based formula. Despite the inclusion of a series of potential exposures that could be related to disease incidence, only family history and the prior development of diabetes-related autoimmunity were significant.

The findings of the present study add evidence to the relationships between atopic diseases and diabetes-related autoimmunity, and also suggest that the interaction depends upon which autoantibody appeared first for eczema but not for asthma or rhinitis.

Acknowledgements A special acknowledgement to the TRIGR families for their participation in this wonderful study and to the dedication of the TRIGR study coordinators who contributed to the amazing success of TRIGR and this ancillary study.

Data availability The data used in the analysis reported in this paper are available from the TRIGR Data Management Unit upon request to the lead author.

Funding This work was supported by the Eunice Kennedy Shriver National Institute of Child Health \& Human Development (NICHD) and the National Institute of Diabetes and Digestive and Kidney Diseases (NIDDK), the National Institutes of Health (NIH) (grant numbers HD040364, HD042444 and HD051997), the Canadian Institutes of Health Research, JDRF and the Commission of the European Communities (specific RTD programme 'Quality of Life and Management of Living Resources', contract number QLK1-2002-00372 'Diabetes Prevention'). Other funding came from the EFSD/JDRF/Novo Nordisk Focused Research Grant, the Academy of Finland (Centre of Excellence in Molecular Systems Immunology and Physiology Research 2012-2017, Decision No. 250114), the Dutch Diabetes Research Foundation and the Finnish Diabetes Research Foundation. Mead Johnson Nutrition provided the blinded colour-coded study formulas.

Authors' relationships and activities The authors declare that there are no relationships or activities that might bias, or be perceived to bias, their work.

Contribution statement All authors attest to meeting ICMJE uniform requirements for authorship by making substantial contributions to the conception and design of this paper; acquisition, analysis and interpretation of the data; drafting or revising the article for intellectual content; and giving final approval of the published version. JPK designed the study, proposed the analysis, interpreted the findings and wrote the manuscript. DC performed the analysis and contributed to the manuscript. SMV, MK and MC designed the study and reviewed/edited the manuscript. JPK and DC are the guarantors of this work and, as such, had full access to all of the data in the study and take responsibility for the integrity of the data and the accuracy of the data analysis. The TRIGR study funder was not involved in the design of the study; the collection, analysis and interpretation of data; writing of the report; or the decision to submit the report for publication.

\section{References}

1. Seaton A, Godden DJ, Brown K (1994) Increase in asthma: a more toxic environment or a more susceptible population? Thorax 49:171-174 
2. Becker $\mathrm{AB}$ (2000) Is primary prevention of asthma possible? Pulmonol 30:63-72

3. Devereux G (2006) The increase in the prevalence of asthma and allergy: food for thought. Nat Rev Immunol 6:869-874

4. Zöllner IK, Weiland SK, Piechotowski I et al (2005) No increase in the prevalence of asthma, allergies, and atopic sensitization among children in Germany: 1992-2001. Thorax 60:545-548

5. Illi S, von Mutius E, Lau S et al (2006) Perennial allergen sensitization early in life and chronic asthma in children: a birth cohort study. Lancet 368(9537):763-770

6. Gough H, Grabenhenrich L, Reich A et al (2015) Allergic multimorbidity of asthma, rhinitis and eczema over 20 years in the German birth cohort MAS. Pediatr Allergy Immunol 26(5):431-437

7. Steck AK, Vehik K, Bonifacio E et al (2015) Predictors of progression from the appearance of islet autoantibodies to early childhood diabetes: The Environmental Determinants of Diabetes in the Young (TEDDY). Diabetes Care 38(5):808-813

8. Ilonen J, Hammais A, Laine A-P et al (2013) Patterns of autoantibody appearance and genetic associations reveal heterogeneous pathways of $\beta$-cell destruction. Diabetes 62(10):3636-3640

9. Krischer JP, Lynch KF, Lernmark Å et al (2017) Genetic and environmental interactions modify the risk of diabetes-related autoimmunity by 6 years of age: The TEDDY Study. Diabetes Care 40(9):1194-1202

10. Wegienka G, Zoratti E, Johnson CC (2015) The role of the earlylife environment in the development of allergic disease. Immunol Allergy Clin N Am 35(1):1-17

11. Virtanen SM (2016) Dietary factors in the development of type 1 diabetes. Pediatr Diabetes 17:S49-S55

12. Osborn DA, Sinn JKH, Jones LJ (2018) Infant formulas containing hydrolysed protein for prevention of allergic disease. Cochrane Database Syst Rev 10:CD003664

13. Knip M, Åkerblom HK, Becker D et al (2014) Hydrolyzed infant formula and early $\beta$-cell autoimmunity: a randomized clinical trial. JAMA 311(22):2279-2287

14. Greer FR, Sicherer SH, Burks AW, Committee on nutrition, section on allergy and immunology (2019) The effects of early nutritional interventions on the development of atopic disease in infants and children: the role of maternal dietary restriction, breastfeeding, hydrolyzed formulas, and timing of introduction of allergenic complementary foods. Pediatrics 143(4):e20190281

15. The TRIGR Study Group (2007) Study design of the Trial to Reduce IDDM in the Genetically at Risk (TRIGR). Pediatr Diabetes 8(3):117-137

16. Åkerblom HK, Knip M, Becker D et al (2007) The TRIGR Study Group. The TRIGR trial: testing the potential link between weaning diet and type 1 diabetes. Immun Endocr Metab Agents Med Chem 7(3): 251-263

17. The TRIGR Study Group (2011) The Trial to Reduce IDDM in the Genetically at Risk (TRIGR) study: recruitment, intervention and follow-up. Diabetologia 54:627-633

18. Sorkio S, Cuthbertson D, Bärlund S et al (2010) Breastfeeding patterns of mothers with type 1 diabetes: results from an infant feeding trial. Diabetes/Metab Res Rev 26(3):206-211

19. Knip M, Virtanen SM, Seppä K et al (2010) Dietary intervention in infancy and later signs of beta-cell autoimmunity. N Engl J Med 363(20):1900-1908

20. ISAAC (2012) ISAAC Phase Three Data. Available from http:/isaac. auckland.ac.nz/phases/phasethree/results/results.php. Accessed 1 Apr 2012

21. Prescott SL, Tang ML (2005) The Australasian Society of Clinical Immunology and Allergy position statement: summary of allergy prevention in children. Med J Aust 182(9):464-467

22. Flöistrup H, Swartz J, Bergström A et al (2006) Allergic disease and sensitization in Steiner school children. J Allergy Clin Immunol 117(1):59-66

23. von Hertzen L, Haahtela T (2005) Signs of reversing trends in prevalence of asthma. Allergy 60(3):283-292

24. Cardwell CR, Shields MD, Carson DJ, Patterson CC (2003) A meta-analysis of the association between childhood type 1 diabetes and atopic disease. Diabetes Care 26(9):2568-2574. https://doi.org/ 10.2337/diacare.26.9.2568

25. Hörtenhuber T, Kiess W, Fröhlich-Reiterer E et al (2018) Asthma in children and adolescents with type 1 diabetes in Germany and Austria: Frequency and metabolic control. Pediatr Diabetes 19(4): 727-732. https://doi.org/10.1111/pedi.12618

26. Black MH, Anderson A, Bell RA et al (2011) Prevalence of asthma and its association with glycemic control among youth with diabetes. Pediatrics 128(4):e839-e847. https://doi.org/10.1542/peds.2010-3636

27. Metsälä J, Lundqvist A, Virta LJ et al (2018) The association between asthma and type 1 diabetes: a paediatric case-cohort study in Finland, years 1981-2009. Int J Epidemiol 47(2):409-416

28. Wahlberg J, Vaarala O, Ludvigsson J, for the ABIS Study Group (2011) Asthma and allergic symptoms and type 1 diabetes-related autoantibodies in 2.5-yr-old children. Pediatr Diabetes 12(7):604-610

Publisher's note Springer Nature remains neutral with regard to jurisdictional claims in published maps and institutional affiliations.

\section{Affiliations}

\section{Jeffrey P. Krischer ${ }^{1}$ (D) David Cuthbertson ${ }^{1}$ (D) $\cdot$ Marisa Couluris $^{2} \cdot$ Mikael Knip $^{3,4}$ (D) Suvi M. Virtanen ${ }^{5,6,7,8}$ (D)}

1 Health Informatics Institute, Morsani College of Medicine, University of South Florida, 3650 Spectrum Boulevard, Suite 100, Tampa, FL 33612, USA

2 Department of Pediatrics, Morsani College of Medicine, University of South Florida, Tampa, FL, USA

3 Children's Hospital, Pediatric Research Center, University of Helsinki and Helsinki University Hospital, Helsinki, Finland

4 Research Program for Clinical and Molecular Metabolism, Faculty of Medicine, University of Helsinki, Helsinki, Finland
5 Unit of Public Health Promotion, National Institute for Health and Welfare, Helsinki, Finland

6 Faculty of Social Sciences/Health Sciences, Tampere University, Tampere, Finland

7 Research, Development and Innovation Center, Tampere University Hospital, Tampere, Finland

8 Tampere Center for Child Health Research, Tampere University Hospital, Tampere, Finland 